\title{
Inequalities for the Tail of the Exponential Series
}

\author{
H. Alzer
}

Abstract. Let

$$
I_{n}(x)=e^{-x}-\sum_{k=0}^{n}(-1)^{k} \frac{x^{k}}{k !}=\sum_{k=n+1}^{\infty}(-1)^{k} \frac{x^{k}}{k !} .
$$

We prove: if $\alpha, \beta>0$ are real numbers and $n \geq 1$ is an integer, then the inequalities

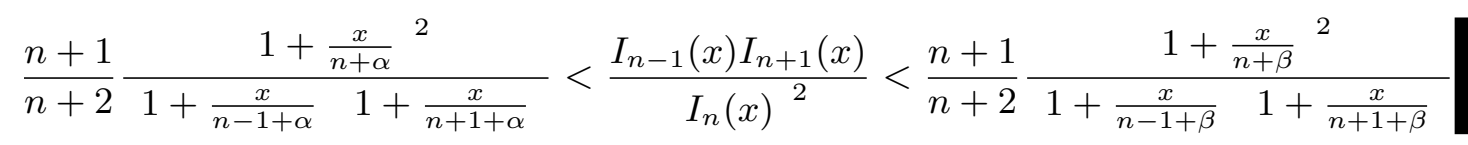

hold for all real numbers $x>0$ if and only if $\alpha \leq 1$ and $\beta \geq 2$. Our result improves inequalities published by M. Merkle in 1997.

Keywords: Exponential function, infinite series, integral means, inequalities, rational approximation

AMS subject classification: 26D15, 33B10

\section{Introduction}

In 1943, P. Kesava Menon [7] proved the inequality

$$
\frac{1}{2}<\frac{J_{n-1}(x) J_{n+1}(x)}{\left(J_{n}(x)\right)^{2}} \quad(x>0 ; n \in \mathbb{N})
$$

where

$$
J_{n}(x)=e^{x}-\sum_{k=0}^{n} \frac{x^{k}}{k !}=\sum_{k=n+1}^{\infty} \frac{x^{k}}{k !}
$$

H. Alzer: Morsbacher Str. 10, D-51545 Waldbröl alzer@wmax03.mathematik.uni-wuerzburg.de

ISSN 0232-2064 / \$2.50 C Heldermann Verlag Berlin 
denotes the tail of the Maclaurin series of the exponential function. Inequality (1.1) can be refined and complemented as

$$
\frac{n+1}{n+2}<\frac{J_{n-1}(x) J_{n+1}(x)}{\left(J_{n}(x)\right)^{2}}<1 \quad(x>0 ; n \in \mathbb{N}) .
$$

Both bounds are sharp (see $[2,6,8])$.

In the recent past, several mathematicians continued the research of inequalities (1.1) and (1.2) and provided different extensions of these results (see [3 - 5, 8 - 11]). Of special interest is a paper of Merkle [10] published in 1997. He presented remarkable properties of $J_{n}(x)$, where $x$ is a negative real number, that is, he investigated

$$
I_{n}(x)=e^{-x}-\sum_{k=0}^{n}(-1)^{k} \frac{x^{k}}{k !}=\sum_{k=n+1}^{\infty}(-1)^{k} \frac{x^{k}}{k !} \quad\left(x>0 ; n \in \mathbb{N}_{0}\right) .
$$

His main result is the following striking companion of (1.2).

Proposition. Let $n \geq 1$ be an integer. Then, for all real numbers $x>0$,

$$
\frac{n}{n+1} \leq \frac{I_{n-1}(x) I_{n+1}(x)}{\left(I_{n}(x)\right)^{2}} \leq \frac{n+1}{n+2} .
$$

Both bounds are best possible.

Moreover, Merkle established the representation

$$
(-1)^{n+1} I_{n}(x)=\frac{x^{n+1}}{(n+1) !\left[1+\frac{x}{n+\theta(n, x)}\right]}
$$

where $\theta(n, x) \in(1,2)$ with $\lim _{x \rightarrow \infty} \theta(n, x)=1$ and

$$
\lim _{x \rightarrow 0^{+}} \theta(n, x)=2 .
$$

An application of (1.4) leads to an additive counterpart of (1.3). If $n \geq 1$ is an integer, then for all $x>0$

$$
0<x^{-2(n+1)}\left[\left(I_{n}(x)\right)^{2}-I_{n-1}(x) I_{n+1}(x)\right]<\frac{1}{(n+1) !(n+2) !}
$$

where both bounds are sharp.

It is not difficult to show that the ratio $\frac{I_{n+1}(x)}{I_{n}(x)}$ can be approximated by linear functions. Indeed, for all integers $n \geq 0$ and real numbers $x>0$ we have

$$
a_{n} x<\frac{I_{n+1}(x)}{I_{n}(x)}<b_{n} x
$$


where the best possible factors (which depend only on $n$ ) are given by $a_{n}=$ $-\frac{1}{n+1}$ and $b_{n}=-\frac{1}{n+2}$. In view of (1.6) it is natural to look for simple rational functions $r_{1}$ and $r_{2}$ such that the double-inequality

$$
r_{1}(x) \leq \frac{I_{n-1}(x) I_{n+1}(x)}{\left(I_{n}(x)\right)^{2}} \leq r_{2}(x)
$$

is valid for all $x>0$ and $n \geq 1$. It is the aim of this paper to show that in fact there exist four quadratic polynomials $p_{1}, p_{2}$ and $q_{1}, q_{2}$ such that (1.7) holds with $r_{1}=\frac{p_{1}}{q_{1}}$ and $r_{2}=\frac{p_{2}}{q_{2}}$. It turns out that our upper and lower bounds for $\frac{I_{n-1}(x) I_{n+1}(x)}{\left(I_{n}(x)\right)^{2}}$ improve those given in (1.3).

\section{Main result}

The following rational approximation to $\frac{I_{n-1}(x) I_{n+1}(x)}{\left(I_{n}(x)\right)^{2}}$ is valid.

Theorem. Let $\alpha, \beta>0$ be real numbers and let $n \geq 1$ be an integer. The inequalities

$$
\begin{aligned}
& \frac{n+1}{n+2} \frac{\left(1+\frac{x}{n+\alpha}\right)^{2}}{\left(1+\frac{x}{n-1+\alpha}\right)\left(1+\frac{x}{n+1+\alpha}\right)} \\
& \quad<\frac{I_{n-1}(x) I_{n+1}(x)}{\left(I_{n}(x)\right)^{2}}<\frac{n+1}{n+2} \frac{\left(1+\frac{x}{n+\beta}\right)^{2}}{\left(1+\frac{x}{n-1+\beta}\right)\left(1+\frac{x}{n+1+\beta}\right)}
\end{aligned}
$$

hold for all real numbers $x>0$ if and only if $\alpha \leq 1$ and $\beta \geq 2$.

Proof. First, we prove: if $0<\alpha \leq 1$ and $\beta \geq 2$, then (2.1) is valid for all $n \geq 1$ and $x>0$. We define for $t>0$

$$
\delta(t, n, x)=\frac{\left(1+\frac{x}{n+t}\right)^{2}}{\left(1+\frac{x}{n-1+t}\right)\left(1+\frac{x}{n+1+t}\right)}
$$

and set $z=n+t>1$. Then we obtain

$$
\frac{\partial \delta(t, n, x)}{\partial t}=2 x \delta(t, n, x) \frac{x^{2}+3 z x+3 z^{2}-1}{z\left(z^{2}-1\right)(x+z)\left((x+z)^{2}-1\right)}>0
$$

which implies that $t \mapsto \delta(t, n, x)$ is strictly increasing on $(0, \infty)$. Thus, it suffices to establish (2.1) for $\alpha=1$ and $\beta=2$.

Taylor's formula yields the integral representations

$$
n !(-1)^{n+1} I_{n}(x)=\int_{0}^{x}(x-t)^{n} e^{-t} d t=x^{n+1} e^{-x} \int_{0}^{1} t^{n} e^{x t} d t .
$$


From (2.2) we conclude that the right-hand side of (2.1) with $\beta=2$ is equivalent to

$$
[f(n-1, x) f(n+1, x)]^{1 / 2}<f(n, x)
$$

where

$$
f(n, x)=\frac{(n+1)(n+2+x)}{n+2} \int_{0}^{1} t^{n} e^{x t} d t .
$$

Inequality (2.3) is a consequence of the stronger inequality

$$
\frac{1}{2}[f(n-1, x)+f(n+1, x)]<f(n, x) .
$$

We prove (2.4) for real numbers $n \geq 1$ and $x>0$. Using

$$
\begin{aligned}
& f(n+1, x)=\frac{(n+2)(n+3+x)}{(n+3) x} e^{x}-\frac{(n+2)^{2}(n+3+x)}{(n+3)(n+2+x) x} f(n, x) \\
& f(n-1, x)=\frac{n+1+x}{n+1} e^{x}-\frac{(n+2)(n+1+x) x}{(n+1)^{2}(n+2+x)} f(n, x)
\end{aligned}
$$

and

$$
\int_{0}^{1} t^{n} e^{x t} d t=\frac{1}{x^{n+1}} \int_{0}^{x} s^{n} e^{s} d s
$$

we obtain

$$
\begin{aligned}
& 2 f(n, x)-f(n-1, x)-f(n+1, x) \\
& \quad=x^{-n-2} u(n, x)\left[\int_{0}^{x} s^{n} e^{s} d s-\frac{v(n, x)}{w(n, x)} x^{n+1} e^{x}\right]
\end{aligned}
$$

where

$$
\begin{aligned}
& u(n, x)=\frac{1}{n+1} x^{3}+\frac{3 n+4}{n+2} x^{2}+\frac{(n+1)(3 n+8)}{n+3} x+(n+1)(n+2) \\
& v(n, x)=x^{2}+\frac{(n+1)(2 n+5)}{n+3} x+(n+1)(n+2) \\
& w(n, x)=x^{3}+\frac{(n+1)(3 n+4)}{n+2} x^{2}+\frac{(n+1)^{2}(3 n+8)}{n+3} x+(n+1)^{2}(n+2) .
\end{aligned}
$$

Let

$$
g(n, x)=\int_{0}^{x} s^{n} e^{s} d s-\frac{v(n, x)}{w(n, x)} x^{n+1} e^{x} .
$$

Partial differentiation leads to

$$
\frac{\partial g(n, x)}{\partial x}=2 \frac{A(n, x)}{(B(n, x))^{2}} x^{n+3} e^{x}
$$


where

$$
\begin{aligned}
A(n, x)= & {\left[n^{2}+5 n+6\right] x^{2} } \\
& +\left[4 n^{3}+22 n^{2}+36 n+18\right] x \\
& +6 n^{4}+44 n^{3}+116 n^{2}+130 n+52 \\
B(n, x)= & {\left[n^{2}+5 n+6\right] x^{3} } \\
& +\left[3 n^{3}+16 n^{2}+25 n+12\right] x^{2} \\
& +\left[3 n^{4}+20 n^{3}+47 n^{2}+46 n+16\right] x \\
& +n^{5}+9 n^{4}+31 n^{3}+51 n^{2}+40 n+12 .
\end{aligned}
$$

Thus, $x \mapsto g(n, x)$ is strictly increasing on $[0, \infty)$. Hence,

$$
g(n, x)>g(n, 0)=0
$$

so that (2.6) - (2.8) imply the validity of inequality (2.4).

Next, we consider the left-hand inequality of (2.1). Let

$$
h(n, x)=(n+1+x) \int_{0}^{1} t^{n} e^{x t} d t .
$$

Applying (2.2) we obtain that the first inequality of (2.1) with $\alpha=1$ is equivalent to

$$
(h(n, x))^{2}<h(n-1, x) h(n+1, x) .
$$

We establish (2.9) for real numbers $n \geq 1$ and $x>0$. Using

$$
\begin{aligned}
& h(n+1, x)=\frac{n+2+x}{x} e^{x}-\frac{(n+1)(n+2+x)}{(n+1+x) x} h(n, x) \\
& h(n-1, x)=\frac{n+x}{n} e^{x}-\frac{(n+x) x}{n(n+1+x)} h(n, x)
\end{aligned}
$$

and (2.5) we obtain

$$
h(n-1, x) h(n+1, x)-(h(n, x))^{2}=\frac{1}{n} x^{-2 n-2} \lambda(n, x) \mu(n, x)
$$

where

$$
\begin{aligned}
& \lambda(n, x)=x^{2}+(2 n+2) x+n^{2}+n \\
& \mu(n, x)=e^{2 x} p(n, x)-e^{x} q(n, x) \int_{0}^{x} s^{n} e^{s} d s+\left(\int_{0}^{x} s^{n} e^{s} d s\right)^{2} \\
& p(n, x)=x^{2 n+1} \frac{(n+x)(n+2+x)}{\lambda(n, x)} \\
& q(n, x)=x^{n} \frac{(n+x)(n+1+x)(n+2+x)}{\lambda(n, x)} .
\end{aligned}
$$


Differentiation gives

$$
\frac{\partial \mu(n, x)}{\partial x}=\frac{b(n, x)}{(\lambda(n, x))^{2}} x^{n-1} e^{x}\left[\frac{a(n, x)}{b(n, x)} x^{n+1} e^{x}-\int_{0}^{x} s^{n} e^{s} d s\right]
$$

where

$$
\begin{aligned}
a(n, x)= & x^{5}+[5 n+4] x^{4} \\
& +\left[10 n^{2}+15 n+4\right] x^{3} \\
& +\left[10 n^{3}+21 n^{2}+8 n\right] x^{2} \\
& +\left[5 n^{4}+13 n^{3}+6 n^{2}-2 n\right] x \\
& +n^{5}+3 n^{4}+2 n^{3} \\
b(n, x)= & x^{6}+[6 n+4] x^{5} \\
& +\left[15 n^{2}+20 n+4\right] x^{4} \\
& +\left[20 n^{3}+40 n^{2}+18 n\right] x^{3} \\
& +\left[15 n^{4}+40 n^{3}+29 n^{2}+4 n\right] x^{2} \\
& +\left[6 n^{5}+20 n^{4}+20 n^{3}+4 n^{2}-2 n\right] x \\
& +n^{6}+4 n^{5}+5 n^{4}+2 n^{3} .
\end{aligned}
$$

Let

$$
\phi(n, x)=\frac{a(n, x)}{b(n, x)} x^{n+1} e^{x}-\int_{0}^{x} s^{n} e^{s} d s
$$

Then

$$
\frac{\partial \phi(n, x)}{\partial x}=2 n \frac{c(n, x)}{(b(n, x))^{2}} x^{n+2} e^{x}
$$

where

$$
\begin{aligned}
c(n, x)= & 6 x^{6}+[36 n+29] x^{5} \\
& +\left[90 n^{2}+137 n+43\right] x^{4} \\
& +\left[120 n^{3}+258 n^{2}+147 n+12\right] x^{3} \\
& +\left[90 n^{4}+242 n^{3}+189 n^{2}+25 n-12\right] x^{2} \\
& +\left[36 n^{5}+113 n^{4}+109 n^{3}+26 n^{2}-6 n\right] x \\
& +6 n^{6}+21 n^{5}+24 n^{4}+9 n^{3} .
\end{aligned}
$$

This implies that $x \mapsto \phi(n, x)$ is strictly increasing on $[0, \infty)$. Hence,

$$
\phi(n, x)>\phi(n, 0)=0
$$

From (2.11) - (2.13) we conclude

$$
\mu(n, x)>\mu(n, 0)=0
$$


so that (2.10) and (2.14) lead to inequality (2.9).

It remains to prove that in (2.1) the parameters $\alpha=1$ and $\beta=2$ are best possible. We assume that there exist numbers $\alpha, \beta>0$ and $n \geq 1$ such that (2.1) is valid for all $x>0$. Since $\lim _{x \rightarrow \infty} \frac{I_{n+1}(x)}{x I_{n}(x)}=-\frac{1}{n+1}$, we obtain from the left-hand side of (2.1) if $x \rightarrow \infty$

$$
\frac{n+1}{n+2} \frac{(n-1+\alpha)(n+1+\alpha)}{(n+\alpha)^{2}} \leq \frac{n}{n+1}
$$

which is equivalent to $\alpha \leq 1$. Applying (1.4) we conclude that the right-hand side of (2.1) is equivalent to

$$
0<\frac{\left(1+\frac{x}{n+\beta}\right)^{2}}{\left(1+\frac{x}{n-1+\beta}\right)\left(1+\frac{x}{n+1+\beta}\right)}-\frac{\left(1+\frac{x}{n+\theta(n, x)}\right)^{2}}{\left(1+\frac{x}{n-1+\theta(n-1, x)}\right)\left(1+\frac{x}{n+1+\theta(n+1, x)}\right)}
$$

Denote herein the right part by $\sigma(n, x, \beta)$. A short computation gives that (1.5) and (2.15) lead to

$$
0 \leq \lim _{x \rightarrow 0^{+}} \frac{\sigma(n, x, \beta)}{x}=\omega(n, \beta)-\omega(n, 2)
$$

where

$$
\omega(n, y)=\frac{2}{n+y}-\frac{1}{n-1+y}-\frac{1}{n+1+y}
$$

Since

$$
\frac{\partial \omega(n, y)}{\partial y}=\frac{2\left[3(n+y)^{2}-1\right]}{(n+y)^{2}(n-1+y)^{2}(n+1+y)^{2}}>0 \quad(y>0)
$$

we conclude that $y \mapsto \omega(n, y)$ is strictly increasing on $(0, \infty)$. Thus, we get from (2.16) that $\beta \geq 2$. This completes the proof of the Theorem

\section{Remarks.}

(1) A simple calculation shows that the inequalities

$$
\begin{aligned}
& \frac{n}{n+1}<\frac{n+1}{n+2} \frac{\left(1+\frac{x}{n+1}\right)^{2}}{\left(1+\frac{x}{n}\right)\left(1+\frac{x}{n+2}\right)} \\
& \frac{n+1}{n+2} \frac{\left(1+\frac{x}{n+2}\right)^{2}}{\left(1+\frac{x}{n+1}\right)\left(1+\frac{x}{n+3}\right)}<\frac{n+1}{n+2}
\end{aligned}
$$

hold for all $n \geq 1$ and $x>0$. Hence, (2.1) (with $\alpha=1$ and $\beta=2$ ) improves the bounds given in (1.3). Moreover, from (2.1) and (2.17) we conclude that inequalities (1.3) are strict. 
(2) Let

$$
\Delta_{n}(a, x)=(n+1) ! \frac{n+a+x}{n+a}\left|I_{n}(x)\right| \quad\left(n \in \mathbb{N}_{0}\right) .
$$

The Theorem yields: if $0<\alpha \leq 1$ and $x>0$, then $n \mapsto \Delta_{n}(\alpha, x)$ is strictly log-convex, whereas, if $\beta \geq 2$ and $x>0$, then $n \mapsto \Delta_{n}(\beta, x)$ is strictly logconcave.

(3) Applying (2.2) we obtain an identity, which connects the functions $I_{n}$ and $J_{n}$ with the integral

$$
\beta_{n}(x)=\int_{-1}^{1} t^{n} e^{-x t} d t
$$

We have

$$
\beta_{n}(x)=\frac{n !}{x^{n+1}}\left[e^{-x} J_{n}(x)-e^{x} I_{n}(x)\right] \quad\left(x>0 ; n \in \mathbb{N}_{0}\right) .
$$

This formula and further properties of $\beta_{n}$ are given in [1: Chapter 5].

Let $A_{n}(x)$ be the arithmetic mean of the function $t \mapsto \exp (x t) \quad(x>0)$ on $[0,1]$ with the weight function $t \mapsto t^{n} \quad\left(n \in \mathbb{N}_{0}\right)$, that is,

$$
A_{n}(x)=\frac{\int_{0}^{1} t^{n} e^{x t} d t}{\int_{0}^{1} t^{n} d t}=(n+1) \int_{0}^{1} t^{n} e^{x t} d t .
$$

The Theorem and (2.2) imply the following integral inequalities.

Corollary. Let $\alpha, \beta>0$ be real numbers and let $n \geq 1$ be an integer. The double-inequality

$$
\frac{\left(1+\frac{x}{n+\alpha}\right)^{2}}{\left(1+\frac{x}{n-1+\alpha}\right)\left(1+\frac{x}{n+1+\alpha}\right)}<\frac{A_{n-1}(x) A_{n+1}(x)}{\left(A_{n}(x)\right)^{2}}<\frac{\left(1+\frac{x}{n+\beta}\right)^{2}}{\left(1+\frac{x}{n-1+\beta}\right)\left(1+\frac{x}{n+1+\beta}\right)}
$$

is valid for all real numbers $x>0$ if and only if $\alpha \leq 1$ and $\beta \geq 2$.

Acknowledgement. I thank the referees for helpful comments. 


\section{References}

[1] Abramowitz, M. and I. Stegun (eds.): Handbook of Mathematical Functions with Formulas, Graphs and Mathematical Tables. New York: Dover 1965.

[2] Alzer, H.: An inequality for the exponential function. Arch. Math. 55 (1990), $462-464$.

[3] Alzer, H., Brenner, J. L. and O. G. Ruehr: Inequalities for the tails of some elementary series. J. Math. Anal. Appl. 179 (1993), 500 - 506.

[4] Chen, W.: Notes on an inequality for sections of certain power series. Arch. Math. 62 (1994), 528 - 530.

[5] Dilcher, K.: An inequality for sections of certain power series. Arch. Math. 60 (1993), $339-344$.

[6] Fink, A. M.: Kolmogorov-Landau inequalities for monotone functions. J. Math. Anal. Appl. 90 (1982), 251 - 258.

[7] Kesava Menon, P.: Some integral inequalities. Math. Student 11 (1943), 36 38.

[8] Merkle, M.: Some inequalities for the chi square distribution function and the exponential function. Arch. Math. 60 (1993), $451-458$.

[9] Merkle, M.: Inequalities for residuals of power series: a review. Univ. Beograd. Publ. Elektrotehn. Fak. Ser. Mat. 6 (1995), 79 - 85.

[10] Merkle, M.: Inequalities for residuals of power expansions for the exponential function and completely monotone functions. J. Math. Anal. Appl. 212 (1997), $126-134$.

[11] Merkle, M. J. and P. M. Vasić: An inequality for residual of Maclaurin expansion. Arch. Math. 66 (1996), $194-196$.

Received 23.07.2001 